\title{
LE MÉCANISME HÉRÉdITAIRE DE LA MALADIE DE DUPUYTREN
}

\author{
par \\ U. Pfändler, La Chaux-de-Fonds (Suisse)
}

\section{CONSIDÉRATIONS GÉNÉRALES}

La contracture de l'aponévrose palmaire, sa limitation à l'auriculaire, à l'annulaire, plus rarement au médius, et le tableau clinique qui en résulte, caractérisent la maladie de Dupuytren. Son conditionnement héréditaire et sa transmission généralement aominante ne sont plus contestés. L'hérédité a été mise en évidence dans $6 \%$ des cas chez Maurer, dans $11 \%$ chez Schnitzler, dans $12 \%$ chez Oehlecker, dans $18 \%$ chez Krogius, dans $27 \%$ chez Kanavel, dans 30\% chez Stackebrandt, et jusqu'à $40 \%$ chez Schröder.

Mais si l'on essaye d'en expliquer le mécanisme héréditaire, on se heurte à de sérieuses difficultés. Quelles sont-elles et d'où proviennent-elles? Sommes-nous en mesure de jeter quelque lumière dans ces secrètes machinations biologiques? Il vaut bien la peine de le tenter; cette maladie est trop fréquente pour que l'on puisse lui méconnaître une importance démographique. Selon les statistiques les plus récentes (Hellmuller, 1948), portant sur 1639 patients atteints, la maladie frappe environ $2 \%$ de la population blanche. On note une participation féminine de $14,5 \%$, tandis que les hommes se taillent la part du lion.

Comme il s'agit d'une hérédité dominante, les fratries atteintes auront nécessairement un parent hétérozygote, à condition qu'une néo-mutation soit exclue. Or l'observation de nombreuses souches a démontré que les femmes transmettent fréquemment la tare sans l'exprimer, tandis que les pères hétérozygotes la manifestent presque toujours. Sa pénétrance est donc très incomplète chez les femmes, mais presque totale chez les hommes. En pathologie héréditaire humaine, les variations de pénétrance vont généralement de pair avec les variations d'expressivité. La maladie de Dupuytren ne fait pas exception. Elle défile sous nos yeux, avec tous ses degrés de manifestation, partant des microsymptômes et aboutissant à l'atteinte grave: les formes légères marquées par la présence de simples nodules dans le creux de la main, les formes moyennes manifestant une contracture du 5 ème, 4 ème, et parfois du $3^{\text {ème }}$ doigt, et les formes graves avec leurs doigts en griffes.

La pénétrance étant moins accentuée chez les femmes, on est d'emblée tenté de présumer qu'il pourrait en être de même pour l'expressivité. Cette conception est confirmée par l'avis de quelques auteurs. Maurer en particulier, pense que les microsymptômes de l'atteinte, représentés par de simples nodules, seraient plus fréquents chez la femme et échapperaient souvent à notre observation. Nous savons par contre que les hommes sont, dans la règle, frappés d'une atteinte plus grave.

Les variations de la pénétrance et de l'expressivité constitueraient, à elles seules, un important obstacle à l'étude du mécanisme héréditaire. Si nous n'étions pas en mesure de les évaluer statistiquement, nous ne pourrions pas déterminer la proportion réelle 


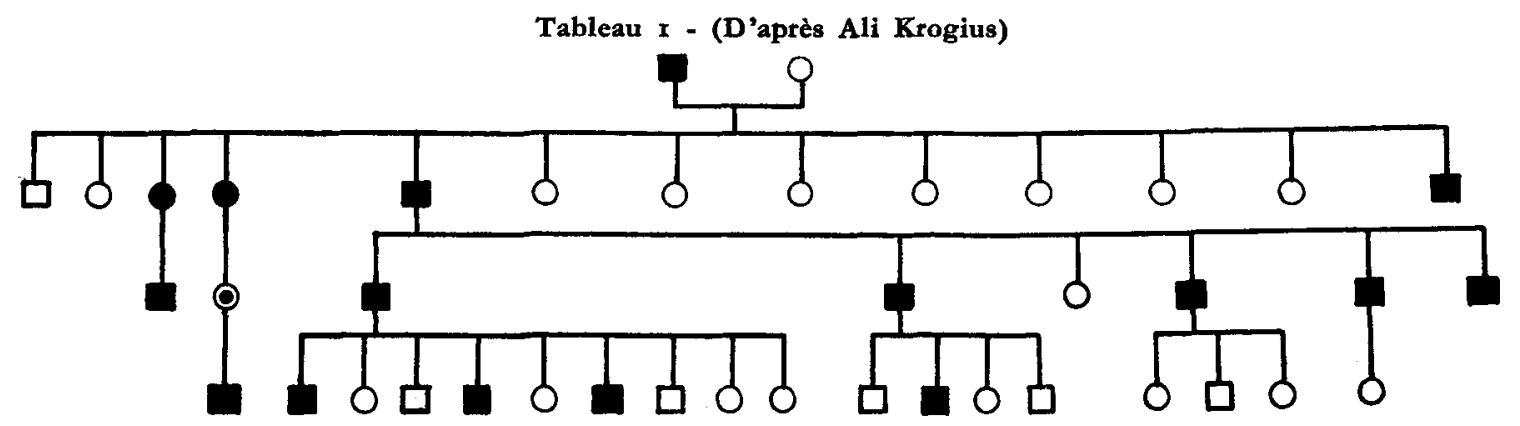

Tableau 2 - (D'après F.R. Jentsch)

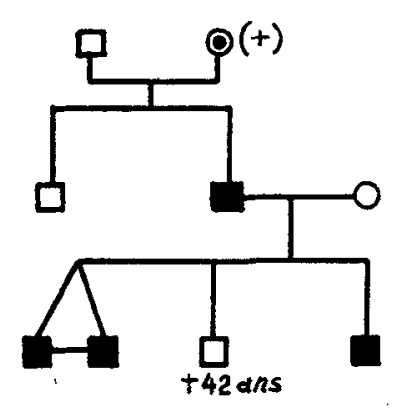

Tableau 3 - (I

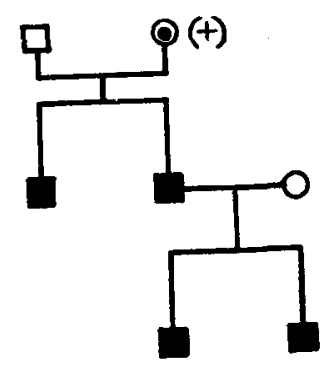

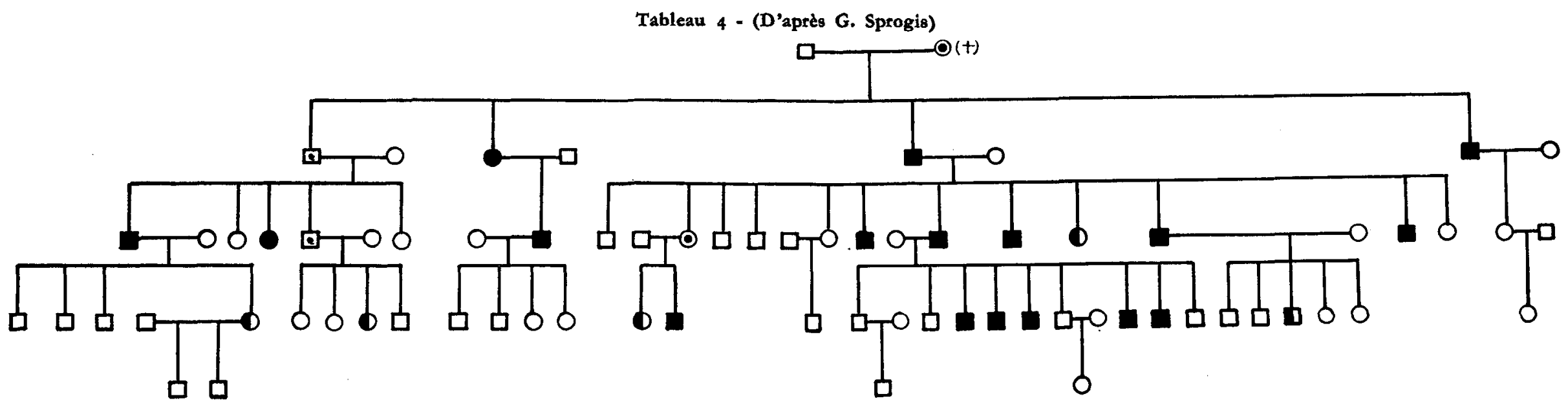

A. Ge. ME. Ge. - Pfändler

https://doi.org/10.1017/S1120962300020667 Published online by Cambridge University Press 
approximative des éléments génotypiquement atteints, et de leurs frères et sœurs normaux. Nous serions alors privés de l'un des critères essentiels de l'hérédité dominante.

Au surplus, d'autres difficultés viennent encore troubler nos investigations. La maladie de Dupuytren n'est pratiquement jamais congénitale. Il existe de nombreux cas juvénils. Mais habituellement, cette atteinte chronique à évolution progressive très lente, apparaît au-delà de 40 ans, pour réaliser son maximum entre 50 et 70 ans. Il en résulte que le dépistage des malades dans les souches atteintes, est rendu singulièrement difficile. Il sera sans autre possible de déterminer les sujets tarés dans les anciennes générations, mais il sera exclu de les reconnaître dans la plus proche descendance qui n'a pas atteint l'âge de manifestation.

A tout cela vient encore s'ajouter un problème de discrimination: il s'agit d'évaluer la part d'influence respective, revenant au patrimoine héréditaire et au milieu. Nous savons que des travaux manuels et des traumatismes surtout chroniques influencent considérablement l'expressivité du gène. Dans ce sens, Gerritzen (1935, cité par Hellmuller), a pratiqué des recherches portant sur 1200 patients atteints de la maladie. Il a observé une fréquence de $4,4 \%$ chez les travailleurs intellectuels, et de $6,4 \%$ chez les travailleurs manuels. Cette proportion indique bien que les influences exogènes se limitent à accentuer l'effet des facteurs héréditaires. Elles peuvent même provoquer la manifestation d'une tare génotypique qui ne se serait jamais exprimée. Mais le patrimoine héréditaire constitue toujours le facteur déterminant par excellence: sans lui, pas de maladie de Dupuytren, exception faite pour des phénocopies toujours possibles.

\section{I - Remarques préliminaires}

Pour expliquer le mécanisme héréditaire de la maladie, nous avons compulsé 24 souches et familles particulièrement typiques de la littérature. Elles frappent d'emblée par l'importante proportion d'éléments atteints; les hommes manifestent la maladie beaucoup plus fréquemment que les femmes. Dès le début, nous partons avec l'idée bien arrêtée que ces souches représentent un matériel sélectionné, marqué par une recrudescence familiale extraordinaire de la tare. Leur taux de pénétrance constitue une valeur réelle pour nos investigations, mais il n'a pas une signification générale. Il est certain que dans une population mélangée, les cas familiaux sont plus rares, et il est probable que la pénétrance soit souvent plus faible. La diminution du taux de pénétrance appelle fréquemment un fléchissement du degré d'expressivité. Dans une population non sélectionnée, les microsymptômes de la maladie sont peut-être plus fréquents que nous ne le pensons. Mais le mécanisme génétique reste le même pour une maladie déterminée, quelles que soient sa pénétrance et son expressivité.

\section{2 - Les 24 souches et familles de la littérature}

= maladie de Dupuytren.

D] = microsymptômes (nodules dans le creux de la main).

$-\quad=$ conducteurs et conductrices de la maladie (ils transmettent le gène taré, sans l'exprimer). 
Acta Geneticae Medicae et Gemellologiae

Tableau 5 - (D'après S. J. Kartschikjan)

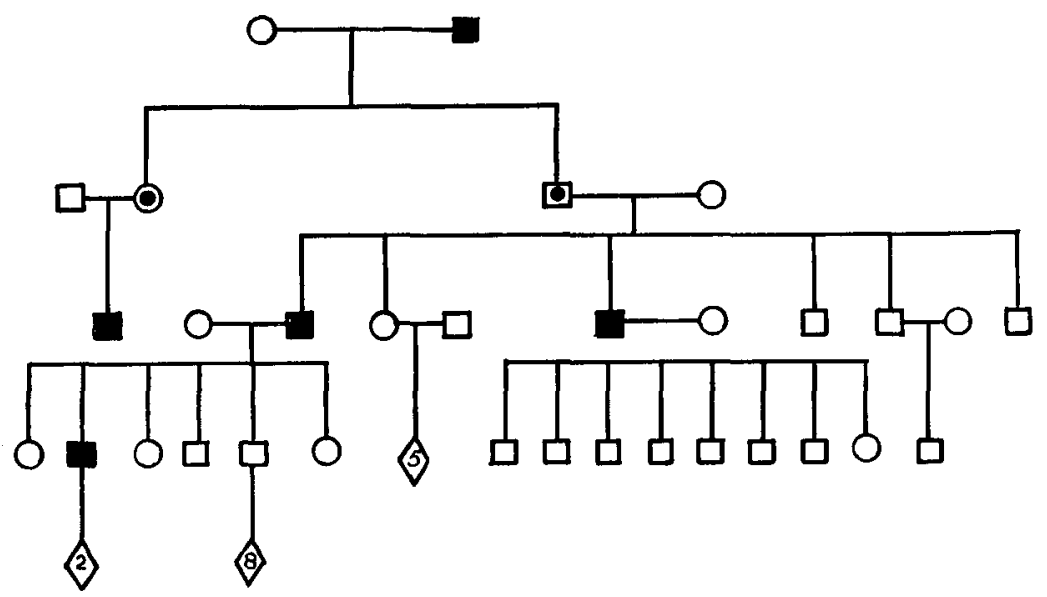

Tableau 6 - (D'après Schröder)

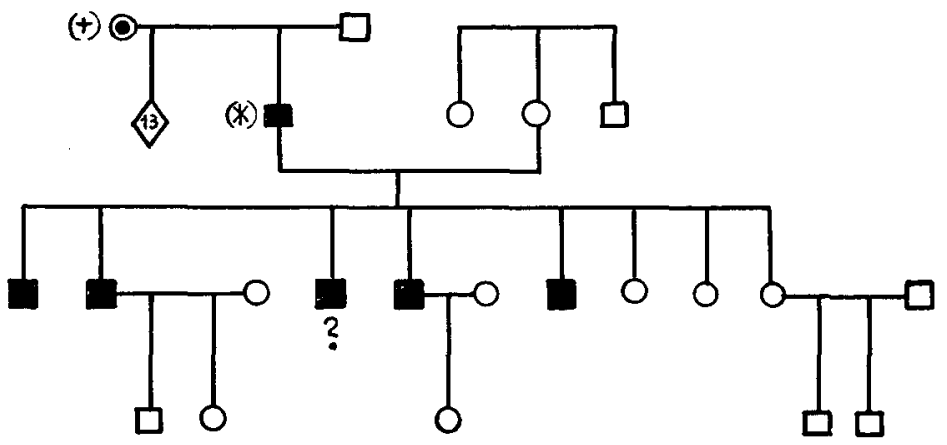

Tableau 7 - (D'après Schröder)

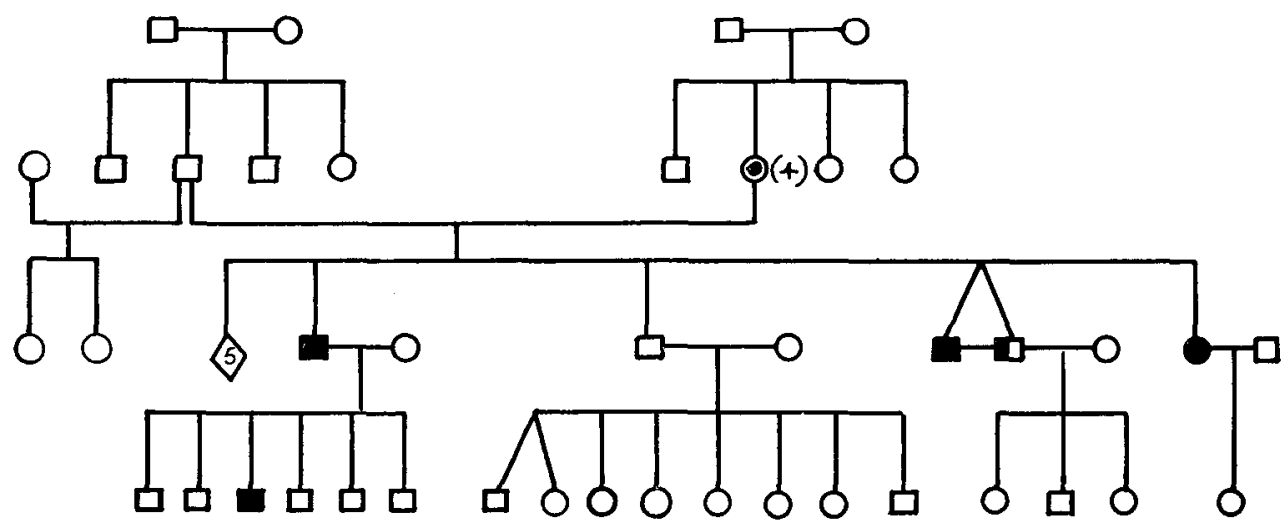

298

https://doi.org/10.1017/S1120962300020667 Published online by Cambridge University Press 


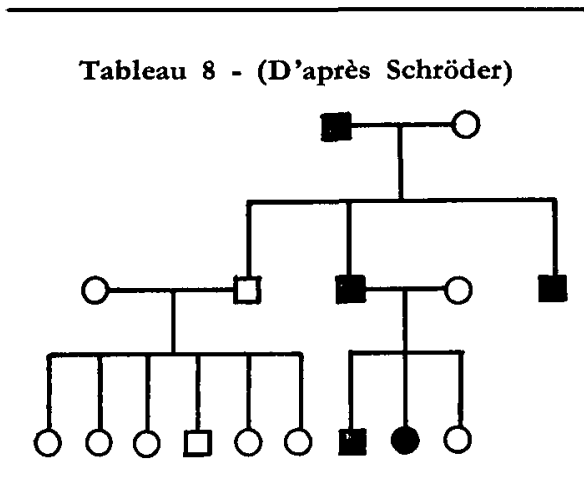

Tableau 9 - (D'après Schröder)
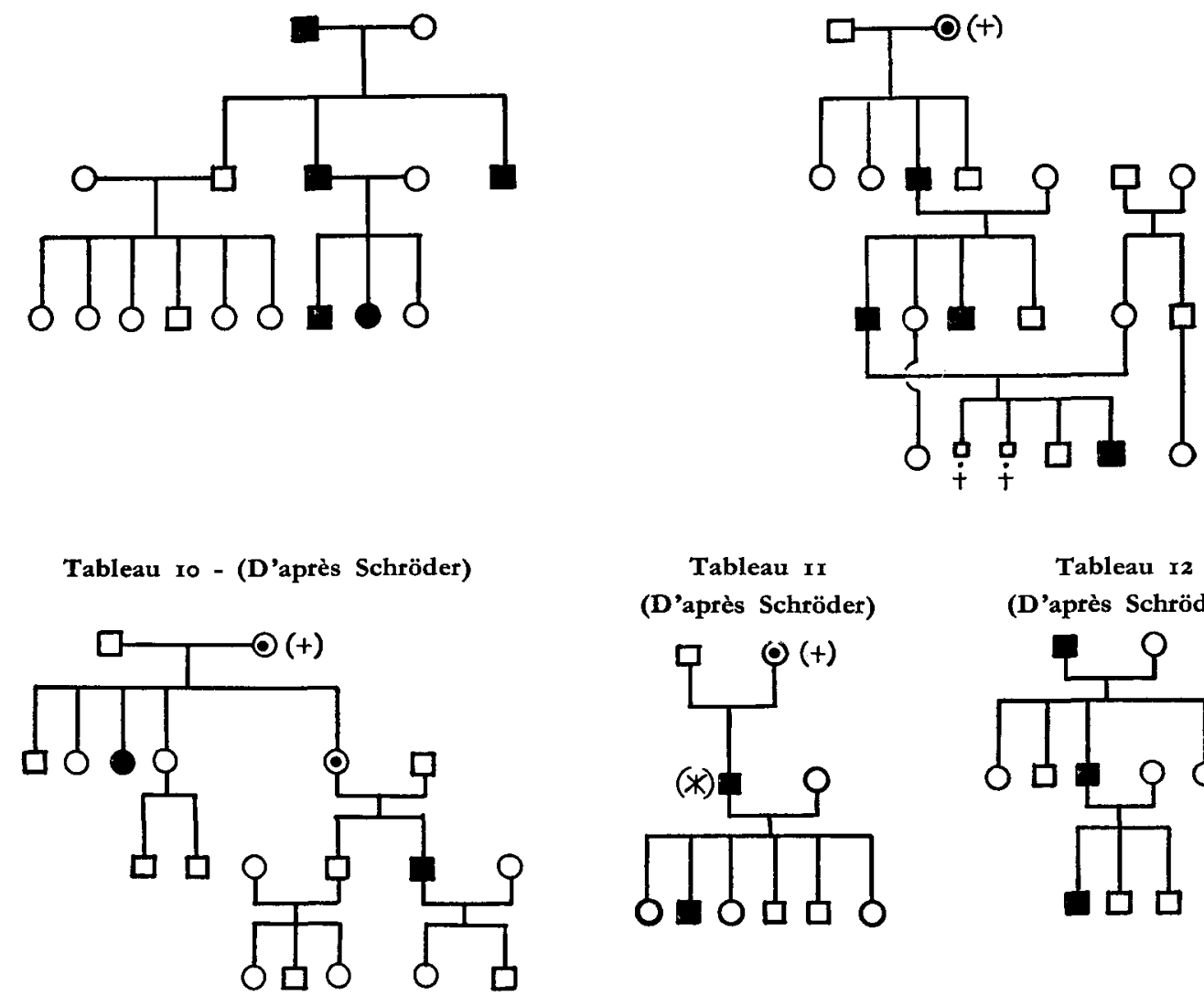

Tableau II (D'après Schröder)

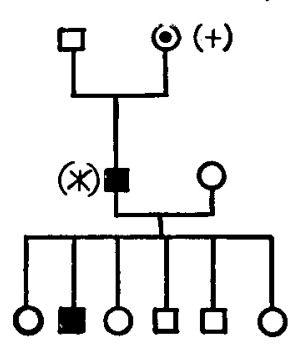

Tableau 12 (D'après Schröder)

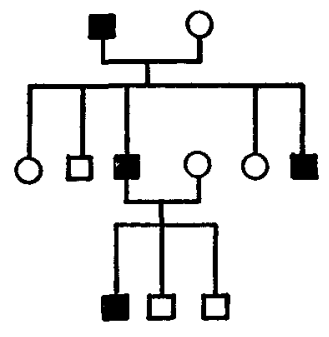

Tableau 13 - (D'après Schröder)

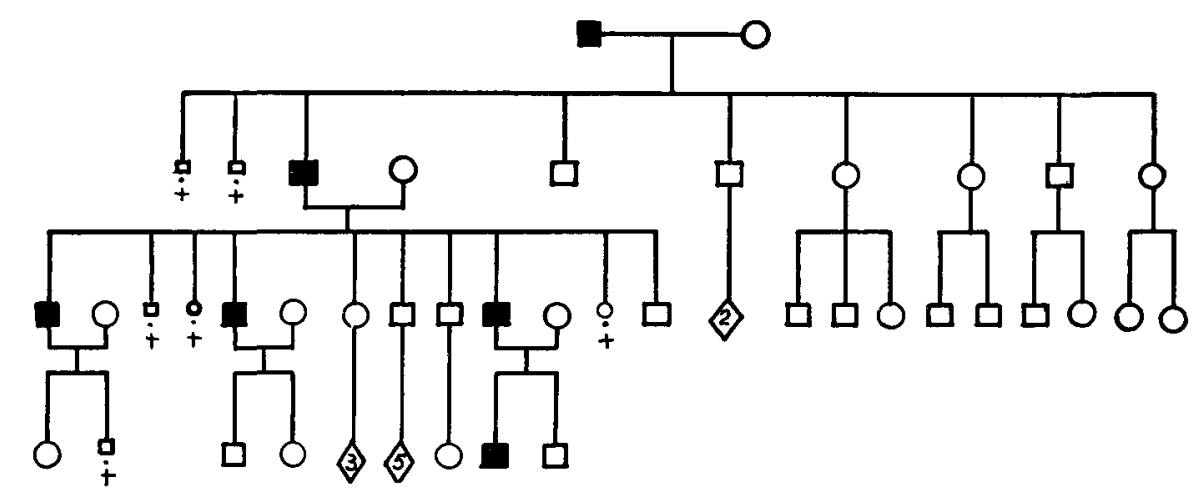




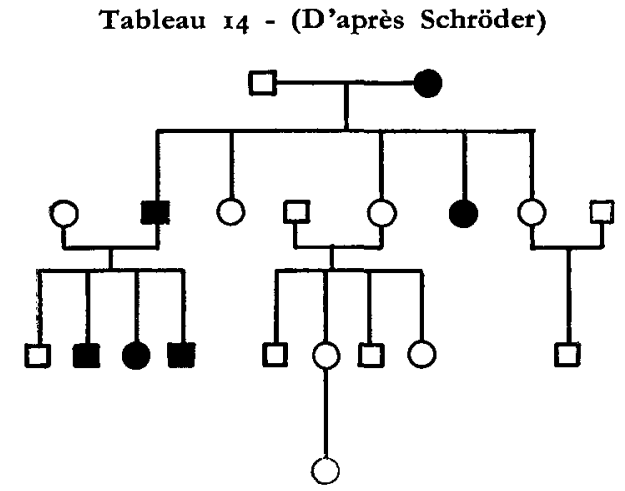

Tableau 16

(D'après Schröder)

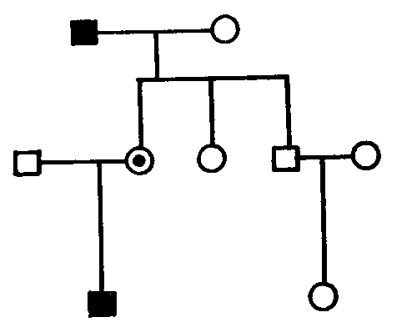

Tableau 17

(D'après K. Deckner)

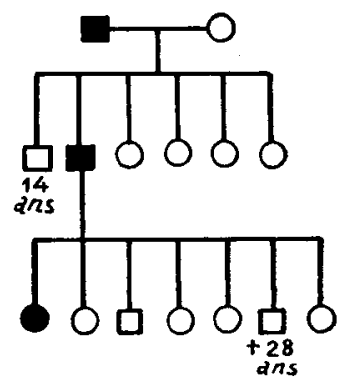

Tableau I5

(D'après Schröder)

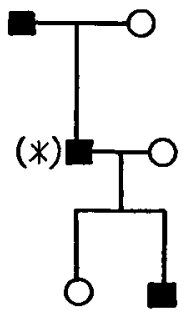

Tableau 18

(D'après H. Stackebrandt)

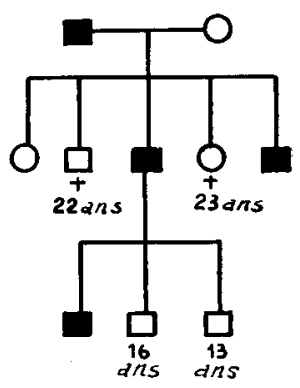

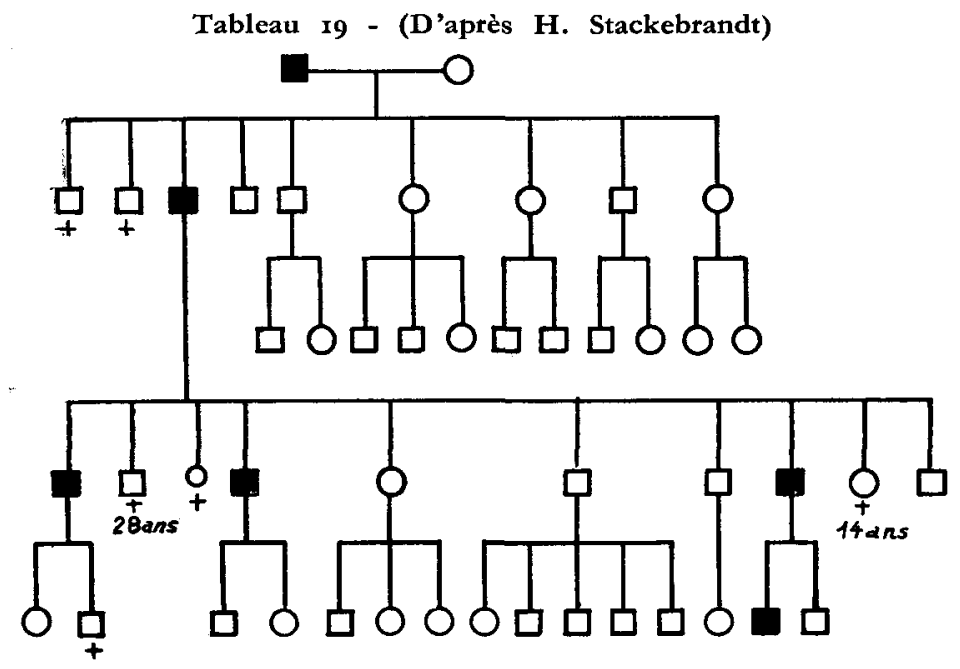

Tableau 20

(D'après H. Stackebrandt

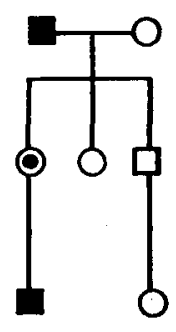


Tableau 2I (D'après H. Stackebrandt)

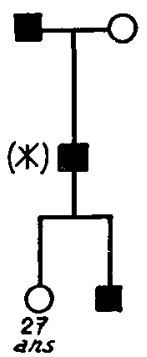

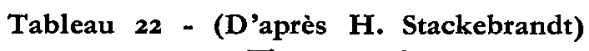

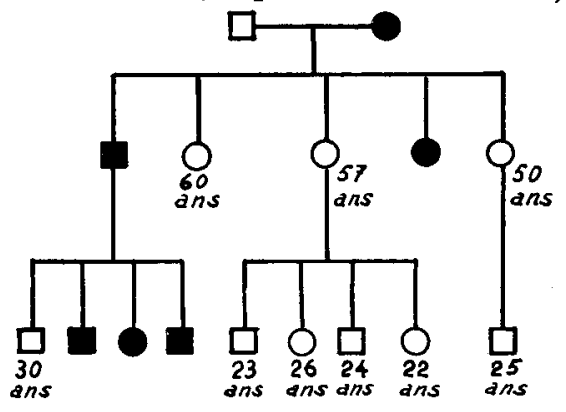

Tableau 23 (D'après Goyrand)

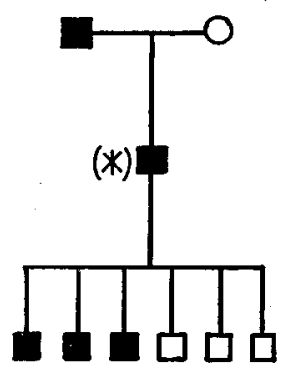

Tableau 24 - (D'après H. Debrunner)

3 - Proportion générale des frères et sœurs malades et normaux, dans les fratries atteintes et dans les fratries saines issues d'un parent malade

Les fratries saines qui n'ont pas atteint l'âge de 20 ans, sont exclues de la statistique.

Les éléments marqués d'un astérisque (*) n'ont pas été comptés, parce que l'on a probablement omis de mentionner leurs frères et sœurs, ou bien parce que le sexe n'a pas été spécifié.

Dans les fratries des souches et familles 1-24, nous trouvons les proportions suivantes:

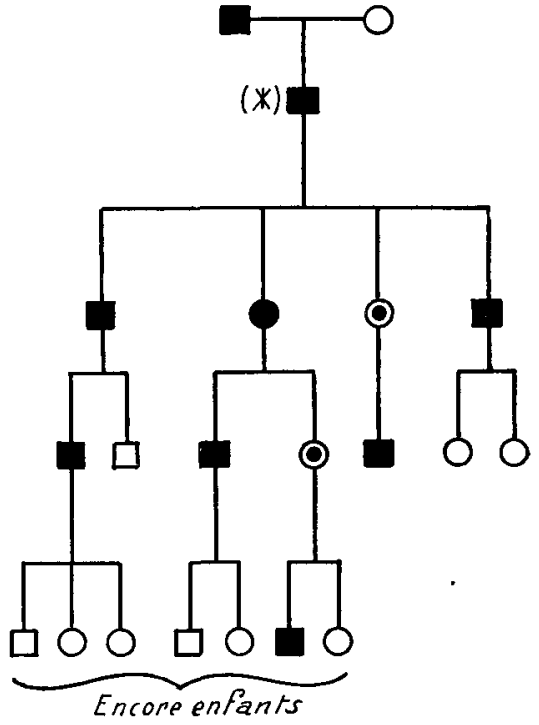

Tableau 25

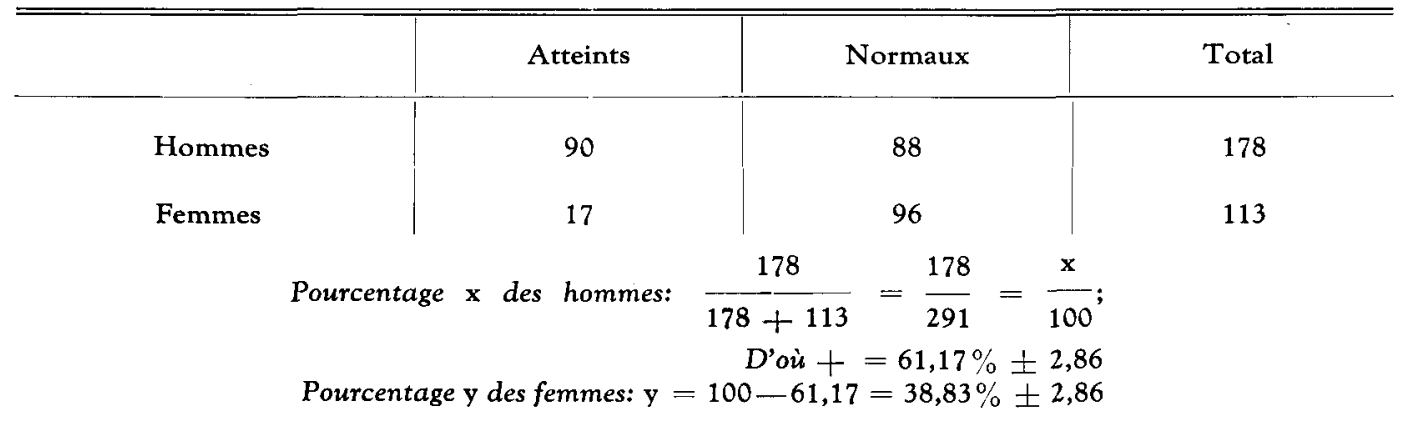




\section{4 - Détermination de la pénétrance du facteur responsable}

Nous disposons en particulier de 3 méthodes différentes pour déterminer la pénétrance:

A) Rechercher la proportion des frères et sœurs atteints et normaux des probands (d'après la méthode de Weinberg).

B) Application d'une méthode directe, en tenant compte du nombre total des enfants, y compris les fratries indemnes provenant de parents atteints.

C) Comparaison du nombre des fratries où l'un des parents est manifestement affecté, avec le nombre total des fratries atteintes.

Avant de porter notre choix sur l'une ou l'autre de ces méthodes, nous voudrions établir un principe qui nous a guidé dans ces investigations, et qui élimine dès le début, tout préjugé en faveur d'un mécanisme génétique déterminé. Nous avons admis sans le moindre doute, la transmission dominante de la maladie de Dupuytren. Mais nous ne savons pas s'il s'agit d'une hérédité autosomique ou gonochromosomique. Etant donné la sexratio de 61,2 hommes pour 38,8 femmes, et l'atteinte prépondérante des hommes (90 17 ), on ne peut pas d'emblée exclure l'existence d'un sexe-linkage incomplet. Pour rechercher la localisation éventuelle du gène dans le segment différencié d'un chromosome sexuel, nous utiliserons une méthode assez connue, dont nous pouvons nous dispenser d'expliquer le fondement. Voici quel est son principe: La somme des enfants atteints du sexe opposé et des normaux du sexe correspondant au grand'parent paternel atteint, divisée par le total des enfants, nous donnera comme résultat $K$. Si ce chiffre est significativement inférieur à $1 / 2$, on peut admettre qu'il s'agit de linkage, et l'on aura en même temps la fréquence du crossing-over $(K)$.

S'il s'agit d'une maladie incomplètement linkée avec le sexe, le facteur taré peut être localisé chez l'homme, soit dans le chromosome $\mathrm{X}$, soit dans le chromosome $\mathrm{Y}$, selon qu'il l'a reçu de sa mère ou de son père. On trouvera donc avant tout des enfants atteints ayant le même sexe que le grand'parent atteint. Il serait par conséquent difficile de vouloir déterminer la pénétrance de la maladie, en se basant sur le comportement de ces fratries. Cette raison nous oblige à écarter d'emblée les méthodes A et B.

Il est cependant possible d'utiliser une méthode modifiée, dont l'exactitude se justifie de la manière suivante: Lorsque le facteur dominant responsable (F) a son siège dans la partie différenciée du gonochromosome, on ne trouvera pas de linkage entre $F$ et le sexe, chez les enfants d'une femme atteinte. Cette dernière ne reçoit le facteur de son père, que lorsqu'il est localisé dans le chromosome X. Nous pouvons en conséquence calculer la pénétrance chez les enfants d'une mère atteinte, puisque chez eux, le linkage est sans effet sur la répartition des éléments atteints selon le sexe.

Cette méthode modifiée nous donne les valeurs suivantes (tableaux 1, 4, 5, 7, $10,14,16,20,22,24)$ :

Tableau 26

\begin{tabular}{l|r|r|r}
\hline \hline $\begin{array}{l}\text { Enfants de mères atteintes ou certai- } \\
\text { nement conductrices de la maladie }\end{array}$ & Atteints & Normaux \\
\hline Hommes & 18 & 6 \\
Femmes & 6 & 13
\end{tabular}


Ces chiffres sont trop petits, pour que l'on puisse en déduire des conclusions probantes. Voyons tout de même ce que nous disent ces résultats.

Si nous admettions une pénétrance complète, nous devrions obtenir :

$$
\begin{aligned}
\frac{18+6}{2} & =12 \text { hommes atteints; } & \chi^{2}=\frac{(18-12)^{2}}{6}=6, \\
\text { et } \frac{6+13}{2} & =9,5 \text { femmes malades; } & \chi^{2}=\frac{(13-9,5)^{2} \times 2}{9,5}=2,579
\end{aligned}
$$

Pour les femmes atteintes, l'écart entre les valeurs théoriques et réelles n'est pas significatif. Par contre le nombre élevé d'hommes réellement atteints ( $\chi^{2}=6=$ significatif), laisse présumer qu'un autre phénomène biologique pourrait intervenir dans la répartition des sexes et des éléments malades. En admettant par exemple une hérédité dominante autosomique avec effet létal électif pour un certain nombre de gamètes $\mathrm{X}$ tarés, on obtiendrait des valeurs pouvant correspondre à peu près à ce tableau. Nous devons donc aussi retenir cette éventualité. Dans ce cas, l'étude de la pénétrance dans les fratries atteintes, devient illusoire; il faut donc écarter également cette méthode modifiée.

La méthode $\mathrm{C}$ que nous utiliserons ici, est la seule dont les valeurs ne soient influencées ni par ce dernier mécanisme, ni par le sexe-linkage incomplet.

\section{MÉTHODE C}

Etant donné que les pères expriment la maladie beaucoup plus fréquemment que les mères de fratries atteintes, nous déterminerons séparément les valeurs dans les deux sexes. C'est-à-dire que nous comparons:

a) Le nombre de fratries où le père est manifestement affecté, avec le nombre total des fratries atteintes ou conductrices certaines, qui ont reçu le gène du père.

b) Le nombre de fratries où la mère est manifestement affectée, avec le nombre total des fratries atteintes ou conductrices certaines, qui ont reçu le gène de la mère.

Il n'est pas possible de dépister les parents hétérozygotes, mais phénotypiquement sains, dont les enfants sont normaux et n'ont pas de descendants malades. Afin de contrebalancer cette source d'erreur, nous éviterons aussi d'enregistrer les parents atteints de fratries saines qui n'ont pas de descendants malades.

Pour une pénétrance de $100 \%$, tous les parents devraient être atteints. Voici la somme des valeurs obtenues dans les tableaux 1-24:

Tableau 27

\begin{tabular}{l|c|c|c}
\hline \hline & Atteints & Normaux & Total \\
\hline Pères & 44 & 3 & 47 \\
Mères & 6 & 8 & 14
\end{tabular}

$$
\begin{aligned}
& \text { D'où } \mathrm{P} 1=\text { pénétrance des pères }=\frac{44}{47}=93,6 \% . \\
& \text { et } \mathrm{P} 2=\text { pénétrance des mères }=\frac{6}{14}=42,9 \% .
\end{aligned}
$$


Il y a $95 \%$ de chance que la pénétrance réelle se situe entre 82,4 et $98,7 \%$ chez les pères, ainsi qu'entre $1.7 ; 7$ et $\cdot 71,1 \%$ chez les mères. L'écart entre les taux de pénétrance $P^{1}$ et $\mathrm{P}^{2}$ nous donne:

\begin{tabular}{r|c|c}
44 & 3 & 47 \\
6 & 8 & 14 \\
\hline 50 & 11 & 61
\end{tabular}

$$
\text { D'où } \chi^{2}=\frac{(334-61 / 2)^{2} \times 61}{50 \times 11 \times 47 \times 14}=15,526
$$

L'écart est donc hautement significatif.

Pour faciliter l'étude du sexe-linkage, il est opportum de savoir quel est le parent hétérozygote probable, dans les fratries atteintes dont le père et la mère sont phénotypiquement sains

\section{5 - Détermination du conducteur probable, lorsque les deux parents d'une fratrie atteinte sont phénotypiquement sains}

Selon le tableau 27, les fratries atteintes ont reçu le facteur 47 fois du père et 14 fois de la mère. Cela nous donne une proportion de $77: 23$, c'est-à-dire qu'il y a trop peu de femmes conductrices.

Ce phénomène peut être en partie imputable a la sélection. La pénétrance de la maladie étant presque totale chez les hommes, mais faible chez les femmes, nous enregistrons plus facilement les fratries ayant reçu le gène du père que de la mère. Ainsi la statistique est faussée. Il est facile de s'en rendre compte, en prenant uniquement la plus ancienne fratrie atteinte de chaque souche. Nous en comptons 24 dans notre matériel statistique.

14 fois, c'est le père qui est atteint, 2 fois c'est la mère qui est atteinte, et 8 fois les deux parents sont phénotypiquement sains. Or dans le tableau 27, nous avons enregistré les deux premières valeurs (14 et 2 ), mais pas la troisième ( 8 pères et 8 mères sains). Nous désirons précisement savoir lequel des partenaires de ces 8 couples parentaux doit être présumé comme conducteur.

La pénétrance chez les hommes est de 93,6\%. Si nous admettons que les 8 pères sont porteurs du gène taré, chacun d'eux aura $100-93,6=6,4 \%$ de chance d'être hétérozygote masqué. Chez les femmes par contre, la pénétrance n'est que $42,9 \%$. Si nous admettons que les 8 femmes de ces couples parentaux renferment le gène invoqué, elles auront chacune $100-42,9=57,1 \%$ de chance de masquer la tare. Les femmes hétérozygotes ont donc $\frac{57,1}{6,4}=$ environ 9 fois plus de chance que les hommes hétérozygotes, de ne pas exprimer le facteur dans leur phénotype. Nous pouvons par conséquent présumer que dans la grande majorité des cas, ce sont les mères qui sont conductrices de ces 8 fratries (ces mères ont été marquées d'une croix $(+)$ dans les tableaux généalogiques). Cette explication paraît tout à fait plausible. L'erreur qui peut en résulter est sans doute minime. 
Nous avons d'ailleurs vu que dans les plus anciennes fratries des 24 souches respectives, 14 pères sont atteints. Mais la pénétrance de la maladie chez les hommes étant de $93,6 \%$, le nombre réel de pères hétérozygotes serait égal à $\frac{14 \times 100}{93,6}=$ environ 15 .

Dans les 8 fratries mentionnées nous aurions alors présumé à tort comme conductrice une femme sur 8 , et il y aurait 15 hommes conducteurs du gène taré au lieu de 14. Cette erreur dont nous faisons ici abstraction, ne doit pas être d'une grande importance pour l'interprétation de la statistique.

En remaniant dans ce sens le tableau 27, nous obtenons:

Tableau 28

\begin{tabular}{l|r}
\hline \hline $\begin{array}{l}\text { Nombre de femmes ayant transmis le gène: } \\
\text { Nombre d'hommes ayant transmis le gène: }\end{array}$ & $14+8=22$ \\
\hline Total: & 47
\end{tabular}

\section{6 - Etude du sexe-linkage incomplet}

La méthode - nous l'avons vu - consiste à déterminer la somme des enfants atteints. du sexe opposé et des normaux du sexe correspondant au grand'parent paternel atteint ou conducteur, divisée par le total des enfants (selon Haldane).

Voici la somme des valeurs obtenues dans les tableaux 1, 2, 3, 4, 5, 6, 7, 8, 9, 10 , $11,12,13,14,15,17,18,19,21,22,23,24$ :

Tableau 29

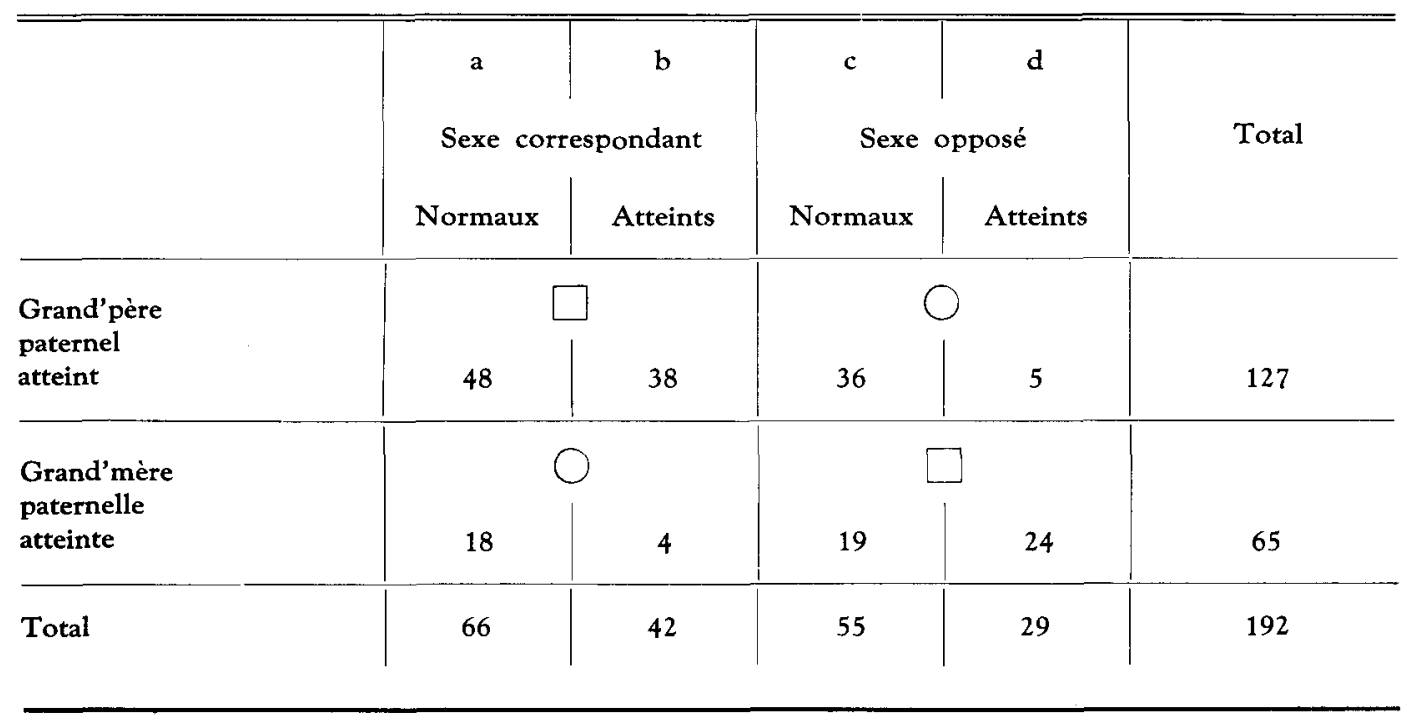


En tout, 14 fratries comptant 65 enfants, ont reçu le gène de la grand'mère paternelle. Le nombre moyen des enfants par fratrie, est donc égal à $\frac{65}{14}=4,643$.

Dans 10 de ces fratries de petits-enfants, aucun des grands'parents n'est atteint, et l'étude des souches ne permet pas de déterminer le conducteur. Mais ces 10 fratries de petits-enfants correspondent précisément aux 8 fratries d'enfants pour lesquelles nous avons présumé les mères comme conductrices (elles sont marquées d'une croix dans les tableaux généalogiques). En admettant qu'une mère sur 8 aurait été reconnue à tort comme hétérozygote masquée, cette dernière représenterait $\frac{10}{8}=1,25$ fratrie de petitsenfants. Or, nous avons vu que chacune de ces fratries aurait en moyenne 4,64 petitsenfants.

$4,64 \times 1,25=5,8$, c'est-à-dire que 6 enfants devraient être soustraits de la deuxième colonne de chiffres horizontale, et additionnés aux valeurs du grand'père paternel atteint (tableau de sexe-linkage N. 29).

Cette attribution erronée de 6 enfants sur un total de 192, est sans doute pratiquement sans grande importance.

Il faut cependant retenir que le nombre d'observations (24 fratries, selon paragraphe 5) est beaucoup trop petit pour que l'on puisse assurer statistiquement la proportion des mères conductrices.

Il serait aussi possible que dans l'une ou l'autre des 8 fratries dont l'ascendance est saine, une nouvelle mutation se soit produite dans un gamète de l'un des parents. Ce dernier serait alors lui-même somatiquement normal.

Nous ne voyons cependant pas de raison majeure qui nous oblige à admettre que ces erreurs d'interprétation possibles, puissent modifier d'une manière importante les répartitions du tableau de sexe-linkage.

Les valeurs sont par contre sérieusement faussées par la pénétrance incomplète de la maladie. Connaissant les taux respectifs pour les deux sexes $\left(P_{1}=93,6\right.$, et $\left.P_{2}=42,9\right)$, nous opérons les rectifications nécessaires et nous obtenons dans le tableau N. 30 les chiffres corrigés. 
Valeurs de linkage corrigées

Tableau 30

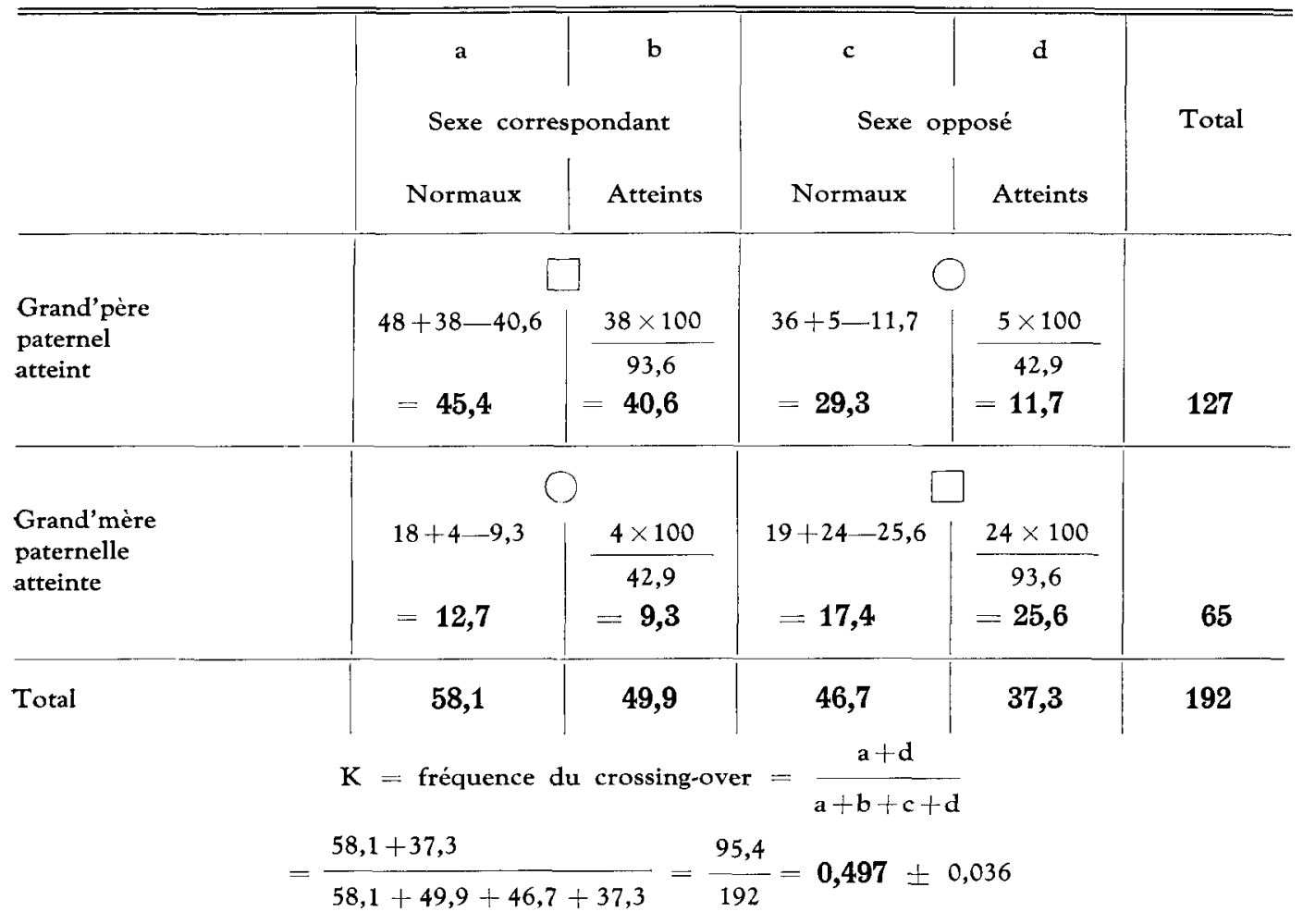

Lorsque $\mathrm{K}=0,5$, il $\mathrm{n}$ 'y a pas de linkage. Si nous tenons compte de l'erreur moyenne $(=0,036)$, la différence entre 0,5 et 0,497 n'est pas probante, et nous écartons l'hypothèse d'un sexe-linkage incomplet.

Nous sommes donc contraint de rechercher une autre explication du mécanisme héréditaire. Il est d'emblée établi que les hommes sont plus fréquemment atteints que les femmes ( 90 hommes pour 17 femmes malades). Mais il faut aussi tenir compte du fait que la sex-ratio dans les fratries atteintes, penche nettement en faveur des hommes $(61,2 \%$ d'hommes et $38,8 \%$ de femmes). A titre de comparaison, voyons comment se comporte la sex-ratio dans les fratries saines dont l'ascendance n'est pas atteinte.

\section{7 - Sex-ratio dans les fratries génotypiquement saines \\ (tableaux 4, 5, 6, 7, 8, 9, 10, 13, 14, 19, 22)}

Nombre d'hommes: 38 Nombre de femmes: 43

Contrairement aux observations faites dans les fratries atteintes, celles qui sont saines ne manifestent pas de sensible différence de pourcentage entre les deux sexes. Le 
chiffre légèrement plus élevé de femmes n'est pas significatif, même si nous admettons la sex-ratio $106 \square$ : $100 \bigcirc$, généralement observée dans la population. Voici d'ailleurs la valeur de $\chi^{2}$ :

Tableau 3 I

\begin{tabular}{l|c|c|c}
\hline \hline & Hommes & Femmes & Total \\
\hline Valeur observée & 38 & 43 & 81 \\
Valeur théorique & 41,68 & 39,32 & 81 \\
$\chi^{2}=\frac{3,68^{2}}{41,68}+\frac{3,68^{2}}{39,32}=\frac{13,54}{41,68}+\frac{13,54}{39,32}=0,324+0,349=0,673$
\end{tabular}

Le $\chi^{2}$ n'étant pas significatif, nous pouvons admettre dans les fratries saines, l'existence d'une proportion sensiblement égale des deux sexes.

Ces valeurs comparées à la sex-ratio dans les fratries atteintes $(38,8 \%$ de femmes), laissent supposer qu'une partie des gamètes $X$ tarés ou des zygotes $X X$ tarés pourraient être détruits, d'autant plus que la proportion générale des femmes atteintes et normales est de $17: 96$ (voir tableau N. 25).

Nous admettons à priori que la maladie de Dupuytren a un conditionnement monogénique, et qu'elle se transmet par voie dominante autosomique. En tenant compte de la pénétrance élective dans les deux sexes, voyons quelles seraient les proportions théoriques d'hommes et de femmes atteints et normaux.

8 - Comparaison des valeurs observées et des taux théoriques, en ce qui concerne la répartition des sujets atteints et normaux dans les deux sexes

P1 $=93,6$

$\mathrm{P} 2=42,9$

$$
\text { Sex-ratio observée: } \frac{\text { Hommes }}{\text { Femmes }}=\frac{178}{113}=\frac{61,2 \%}{38,8 \%}
$$

a) Chez les hommes, le taux théorique d'éléments phénotypiquement atteints pour une hérédité dominante, serait:

$$
\frac{178 \times 93,6}{2 \times 100}=83,3
$$

D'où la proportion des hommes sains $=178-83,3=94,7$. 
Comparons ces chiffres aux valeurs observées:

\begin{tabular}{l|c|c|c}
\multicolumn{3}{c}{ Tableau 32 } \\
\hline \hline & $\begin{array}{c}\text { Hommes } \\
\text { atteints }\end{array}$ & $\begin{array}{c}\text { Hommes } \\
\text { sains }\end{array}$ & Total \\
\hline Valeur observée & 90 & 88 & 178 \\
Valeur théorique & 83,3 & 94,7 & 178 \\
$\qquad \chi^{2}=\frac{6,7^{2}}{83,3}+\frac{6,7^{2}}{94,7}=0,539+0,474=1,013$
\end{tabular}

Il n'existe pas d'écart significatif entre les valeurs observées et théoriques. serait de

b) Chez les femmes, le taux théorique d'éléments phénotypiquement atteints,

$$
\frac{113 \times 42,9}{2 \times 100}=24,2
$$

D'où la proportion de femmes saines $=113-24,2=88,8$.

Comparons à nouveau les chiffres théoriques avec les valeurs observées:

Tableau 33

\begin{tabular}{l|c|c|c}
\hline \hline & $\begin{array}{c}\text { Femmes } \\
\text { atteintes }\end{array}$ & $\begin{array}{c}\text { Femmes } \\
\text { saines }\end{array}$ & Total \\
\hline Valeur observée & 17 & 96 & 113 \\
Valeur théorique & 24,2 & 88,8 & 113
\end{tabular}

$$
\chi^{2}=\frac{7,2^{2}}{24,2}+\frac{7,2^{2}}{88,8}=2,142+0,583=2,725
$$

Le $\chi^{2}$ n'est pas significatif. Le surplus considérable de femmes phénotypiquement saines provient en premier lieu de la pénétrance incomplète du gène responsable. 
9 - Proportion générale corrigée des frères et sœurs atteints et normaux

Dans le tableau N. 25, nous n'avons pas tenu compte des taux de pénétrance pour les deux sexes $\left(\mathrm{P}^{1}=93,6\right.$ et $\left.\mathrm{P}^{2}=42,9\right)$. Voici les valeurs obtenues, en opérant les corrections nécessaires:

Tableau 34

\begin{tabular}{|c|c|c|c|c|c|c|}
\hline & Att & & Norm & laux & & Total \\
\hline \multirow{2}{*}{ Hommes } & $90 \times 100$ & $a$ & $178-96,1$ & $=$ & $b$ & \\
\hline & \multicolumn{2}{|c|}{96,1} & \multicolumn{3}{|c|}{81,9} & 178 \\
\hline \multirow{2}{*}{ Femmes } & $17 \times 100$ & c & $113-39,6$ & $=$ & $d$ & \\
\hline & \multicolumn{2}{|c|}{39,6} & \multicolumn{3}{|c|}{73,4} & 113 \\
\hline
\end{tabular}

Nous allons vérifier si, pour l'éventualité d'une dominance régulière, il existe une différence significative entre les valeurs $a$ et $b, a$ et $c, b$ et $d, c$ et $d$.

A) Valeur de $\chi^{2}$ pour $a-b$.

Tableau 35

\begin{tabular}{l|c|c|c}
\hline \hline & Hommes atteints & Hommes normaux & Total \\
\hline Observation & 96,1 & 81,9 & 178 \\
Théorie & 89 & 89 & 178 \\
& $\chi^{2}=\frac{7,1^{2}}{89}+\frac{7,1^{2}}{89}=2\left(\frac{50,41}{89}\right)=2 \times 0,566=1,132$ &
\end{tabular}

Le $\chi^{2}$ n'est pas significatif.

B) Valeur de $\chi^{2}$ pour $a-c$.

Tableau 36

\begin{tabular}{l|c|c|c}
\hline \hline & Hommes atteints & Femmes atteintes & Total \\
\hline Observation & 96,1 & 39,6 & 135,7 \\
Théorie & 67,85 & 67,85 & 135,7 \\
& $\chi^{2}=\frac{28,25^{2}}{67,85}+\frac{28,25^{2}}{67,85}=2\left(\frac{798,06}{67,85}\right)=2 \times 11,76=23,52$
\end{tabular}

Le $\chi^{2}$ est hautement significatif. 
C) Valeur de $\chi^{2}$ pour $b-d$.

Tableau 37

\begin{tabular}{l|c|c|c}
\hline \hline & Hommes normaux & Femmes normales & Total \\
\hline Observation & 81,9 & 73,4 & 155,3 \\
Théorie & 77,65 & 77,65 & 155,3 \\
& $\chi^{2}=\frac{4,25^{2}}{77,65}+\frac{4,25^{2}}{77,65}=2\left(\frac{18,06}{77,65}\right)=2 \times 0,232=0,464$ &
\end{tabular}

Le $\chi^{2} n^{\prime}$ est pas significatif.

D) Valeur de $\chi^{2}$ pour $c-d$.

Tableau 38

\begin{tabular}{l|c|c|c}
\hline \hline & Femmes atteintes & Femmes normales & Total \\
\hline Observation & 39,6 & 73,4 & 113 \\
Théorie & 56,5 & 56,5 & 113 \\
& $\chi^{2}=\frac{16,9^{2}}{56,5}+\frac{16,9^{2}}{56,5}=2\left(\frac{285,61}{56,5}\right)=2 \times 5,055=10,11$ &
\end{tabular}

Le $\chi^{2}$ est hautement significatif.

Les valeurs $A, B, C, D$, permettent de conclure qu'il se forme trop peu de femmes tarées, en comparaison des femmes saines et des hommes atteints.

\section{Io - Une nouvelle théorie du mécanisme héréditaire}

L'analyse statistique des souches et des familles $1-24$, nous amène tout naturellement à formuler une nouvelle théorie pour l'hérédité de la maladie de Dupuytren.

Une étude pleine d'intérêt, portant sur 19164 cas, et publiée en 1952 par Jalavisto, traite le problème de la vitalité des gamètes $\mathrm{X}$ et $\mathrm{Y}$. Voici les conclusions essentielles de ce travail:

"Il naît actuellement davantage de garçons et moins de filles qu'autrefois. Bien que les fausses-couches et les morts-nés puissent influencer la proportion des sexes, nous devons tout de même admettre que la sex-ratio primaire accuse un important excédent de garçons. Chez les enfants nés d'une fécondation artificielle, 60 à $70 \%$ sont des mâles. On a en outre observé que la proportion des garçons est beaucoup plus élevée, lorsque la mère a subi une fécondation artificielle extraconjugale. Ce phénomène s'explique sans doute par le fait que, dans ces cas-là, la période s'écoulant entre l'éjaculation et la fécondation est plus longue. Par contre si c'est le mari lui-même qui fournit le sperme, on peut l'injecter immédiatement à la mère, et la perte de temps est plus courte.

Se basant sur ces observations et sur les données de la littérature, Jalavisto avance 
l'hypothèse que les gamètes femelles $X$ du père seraient plus sensibles aux influences péristatiques, et plus facilement détruits que les gamètes $Y$. Lorsque l'intervalle entre l'éjaculation et l'ovulation augmente, la chance d'obtenir une fille diminuerait. La sex-ratio serait dépendante de la fréquence des cohabitations. Celles-ci seraient plus rapprochées dans les familles nombreuses; la probabilité que le gamète maternel soit fécondé au moment de l'ovulation serait alors plus grande, et la proportion des filles augmenterait. L'étonnant excédent de mâles pendant les périodes de guerre, s'expliquerait par le fait que les congés militaires ne sont pas en corrélation temporelle avec l'ovulation. Ainsi la probabilité d'une incidence entre le coït et l'ovulation serait plus faible. De même, l'emploi accru de moyens anticonceptionnels, pourrait détruire de préférence les gamètes $\mathrm{X}$ du père, et favoriser la naissance de garçons $"$.

Cette théorie présente un grand intérêt pour nos investigations. Elle établit le principe d'une plus grande vulnérabilité des gamètes $X$.

Nous exposerons d'emblée le sens de notre hypothèse, et nous la vérifierons ensuite sur la base des valeurs statistiques. Nous admettons évidemment que la maladie de Dupuytren se transmet par voie dominante simple. Le facteur en question serait donc localisé dans un autosome. Nous nommerons Gamète $X$ taré, la cellule sexuelle femelle qui porte le gène responsable, et Gamète $\mathrm{Y}$ taré, la cellule mâle qui renferme le facteur invoqué. Enfin, pour expliquer l'excédent de mâles et la faible proportion de femmes atteintes, nous admettons que le facteur responsable de la tare, serait létal pour une partie des zygotes femelles XX. Les zygotes XY tarés seraient par contre parfaitement viables. De nombreux calculs nous ont permis de fixer empiriquement la fréquence de la létalité pour les zygotes XX tarés.

Nous estimons qu'environ $60 \%$ des cellules $X$ tarées auraient une répulsion pour le gamète $X$ sain.

Que se passera-t-il, si nous supposons ce phénomène? Les cellules sexuelles $\mathrm{Y}$ malades du père, rencontreront un ovule $(\mathrm{X})$ normal, et l'œuf sera généralement viable. Les spermatozoïdes $\mathrm{X}$ tarés rencontreront nécessairement un ovule $\mathrm{X}$ sain. Mais dans $60 \%$ des cas, l'association sera létale pour le zygote. $40 \%$ de ces œufs seraient viables.

Les choses se passent différemment, lorsque c'est la mère qui fournit le gamète $\mathrm{X}$ taré. Celle-ci formera 50\% d'ovules normaux, et 50\% de cellules renfermant le facteur morbide. De ces dernières, la moitié seront fécondées par un spermatozoide $Y$, et le zygote sera viable. L'autre moitié sera fécondée dans $40 \%$ des cas, c'est-à-dire $\frac{40 \times 25}{100}=10$ fois par un gamète paternel $\mathrm{X}$ sain, et le zygote sera, lui aussi, viable. Il reste $60 \%$ d'ovules $X$ tarés, c'est-à-dire $\frac{60, \times 25}{100}=15$ cellules dont l'association avec un spermatozoïde $X$ sain, est incompatible. Mais comme les ovules tarés se trouvent aussi en présence de gamètes Y sains, ces derniers les féconderont électivement. Ainsi nous obtenons un excédent de mâles qui remplacent les femelles prévues par le hasard des combinaisons mendéliennes.

Nous admettons en principe une sex-ratio normale de $1: 1$, tout en sachant que la proportion générale des sexes est estimée à $106 \square: 100 \bigcirc$. Mais dans les fratries saines de 
notre matériel sélectionné, nous comptons 38 hommes pour 43 femmes. Les observations sont trop peu nombreuses pour que l'on puisse préciser davantage la proportion des sexes. De toute manière, nous ne savons pas quelle est exactement la sex-ratio primaire dans nos fratries atteintes.

Avec une sex-ratio de $1: 1$, et une incompatibilité essentielle pour $60 \%$ des zygotes $\mathrm{XX}$ tarés, nous obtenons théoriquement la descendance suivante dans les fratries atteintes, issues d'un père ou d'une mère hétérozygote:

a) Les mères hétérozygotes forment:

$50 \%$ de gamètes $X$ sains, et $50 \%$ de gamètes $X$ tarés, dont $60 \%$, c'est-à-dire $\frac{50 \times 60}{100}=30$ ont une aversion pour le spermatozoïde $\mathrm{X}$ sain, et donnent des hommes tarés.

Il restera donc $50-30=20 \%$ de gamètes $X$ tarés.

Mais les femmes ne fourniront le gène morbide que dans $\frac{22}{69}=32 \%$ des cas (voir tableau N. 28).

$100-32=68$ fois sur 100 , c'est le père qui est hétérozygote.

Les mères hétérozygotes produiront donc:

$$
\begin{aligned}
& \frac{50 \times 32}{100}=16 \times \text { sains, } \\
& \frac{20 \times 32}{100}=6,4 \times \text { tarés, } \\
& \frac{30 \times 32}{100}=9,6 \times \text { tarés qui donnent des mâles. }
\end{aligned}
$$

b) Les pères hétérozygotes forment:

$25 \%$ de gamètes $Y$ sains,

$25 \%$ de gamètes $\mathrm{Y}$ tarés,

$25 \%$ de gamètes $X$ sains, et

$25 \%$ de gamètes $X$ tarés, dont $60 \%$, c'est-à-dire $\underline{25 \times 60}=15$ sont détruits.

11 reste donc $25-15=10$ gamètes $X$ tarés. $\quad 100$

Comme les pères fournissent le gamète taré dans $68 \%$ des cas, ils produiront:

$$
\begin{aligned}
& \frac{25 \times 68}{100}=17 \mathrm{Y} \text { sains, } \\
& \frac{25 \times 68}{100}=17 \mathrm{Y} \text { tarés } \\
& \frac{25 \times 68}{100}=17 \times \text { sains, et } \\
& \frac{10 \times 68}{100}=6,8 \times \text { tarés. }
\end{aligned}
$$

21 - A. Ge. Me. Ge (IV). 
Mais une partie des gamètes $\mathrm{X}$ tarés sont détruits. De ce fait, la répartition $17+17+$ $+17+6,8$ des gamètes provenant des pères hétérozygotes, ne représente que le $57,8 \%$ des transmissions, tandis que les pères fournissent en réalité le $68 \%$ des cellules sexuelles.

Ainsi la perte d'un certain nombre de $\mathrm{X}$ tarés, provoque un changement dans la proportion des gamètes. En réalité, les pères restent tout de même transmetteurs de gènes dans $68 \%$ des cas. Nous devons par conséquent augmenter les valeurs 17, 17, 17, et 6,8 dans la proportion $68: 57,8$.

Cela nous donne pour les pères hétérozygotes, la répartition suivante des gamètes:

$$
20+20+20+8=68
$$

A) Les gamètes des mères donneront:

$$
\begin{aligned}
\frac{16}{2} & =8 \text { hommes sains, } \\
\frac{16}{2} & =8 \text { femmes saines, } \\
\frac{6,4}{2} & =3,2 \text { hommes tarés, et } \\
\frac{6,4}{2} & =3,2 \text { femmes tarées, auxquels viennent encore s'ajouter } \\
& =9,6 \text { hommestarés }
\end{aligned}
$$

B) Les gamètes des pères donneront:

20 hommes sains,

20 femmes saines,

20 hommes tarés, et

8 femmes tarées.

Total A+B:

\begin{tabular}{ll|ll}
$8+20$ & $=$ & 28 hommes sains, \\
$8+20$ & $=$ & 28 femmes saines, \\
$3,2+8$ & $=$ & 11,2 femmes tarées, et \\
$3,2+9,6+20$ & $=$ & 32,8 hommes tares.
\end{tabular}

Total 100

Nous obtenons ainsi les proportions théoriques suivantes:

Tableau 39

\begin{tabular}{c|c|c|c}
\hline \hline & Atteints & Normaux & Total \\
\hline Hommes & $32,8 \%$ & $28 \%$ & $60,8 \%$ \\
Femmes & $11,2 \%$ & $28 \%$ & $39,2 \%$ \\
\hline Total & $44 \%$ & $56 \%$ & $100 \%$
\end{tabular}


Nous allons vérifier s'il existe un écart significatif entre les valeurs théoriques du tableau N. 39, et la répartition corrigée pour une pénétrance incomplète, des 291 cas réellement observés, qui figurent au tableau N. 34. Dans ce but, nous répartissons les 291 cas. selon les proportions du tableau N. 39. Cela nous donne:

Tableau 40

\begin{tabular}{|c|c|c|c|}
\hline & Atteints & Normaux & Total \\
\hline \multirow{2}{*}{ Hommes } & $291 \times 32,8$ & $291 \times 28$ & \\
\hline & $100 \quad 95,4$ & 100 & 176,9 \\
\hline \multirow{2}{*}{ Fermmes } & $291 \times 11,2$ & $291 \times 28$ & \\
\hline & 32,6 & 100 & 114,1 \\
\hline Total & 128 & 163 & 291 \\
\hline
\end{tabular}

Afin de vérifier l'écart entre les chiffres observés (tableau 34) et théoriques (tableau 40), nous appliquons la méthode du $\chi^{2}$ avec trois degrés de liberté, en comparant chaque valeur du tableau 34 avec celles du tableau 40 . Nous obtenons ainsi:

$$
\begin{aligned}
& \chi^{2}=\frac{(96,1-95,4)^{2}}{95,4}+\frac{(39,6-32,6)^{2}}{32,6}+\frac{(81,9-81,5)^{2}}{81,5}+\frac{(73,4-81,5)^{2}}{81,5} \\
& =0,005+1,503+0,002+0,805=2,315
\end{aligned}
$$

Le $\chi^{2}$ n'est pas significatif.

\section{Conclusion}

La répartition observée et corrigée des femmes et des hommes atteints et normaux (tableau N. 34), comparée aux prévisions théoriques (tableau N. 40), ne présente pas d'écart significatif. Dans les valeurs réelles, nous obtenons une proportion légèrement trop élevée de femmes atteintes, au détriment des femmes normales. Cette différence peut provenir d'une erreur dans l'estimation du taux de pénétrance. Ce dernier ne se base en effet que sur 14 observations (voir tableau N. 27).

L'étonnante concordance relative des valeurs théoriques et réelles, rend plausible notre hypothèse invoquée pour expliquer le mécanisme héréditaire.

La maladie se transmet par voie dominante autosomique. Le facteur responsable serait létal pour $60 \%$ des zygotes XX tarés. Lorsque le père forme les cellules sexuelles $\mathrm{X}$ malades, $60 \%$ des zygotes seraient définitivement perdus. Si par contre, c'est la mère qui produit les gamètes $\mathrm{X}$ tarés, $60 \%$ d'entre eux, atteints d'une incompatibilité 
essentielle pour le spermatozoïde $X$ sain, seraient fécondés électivement par un gamète Y normal. Ainsi ils échapperaient à la destruction. Ce mécanisme nous expliquerait fort bien l'excédent d'hommes malades, la faible proportion de femmes atteintes, et la sexratio de $61,2: 38,8$.

\section{Résumé}

La maladie de Dupuytren est plus fréquente qu'on ne le pense généralement.

Nous soumettons à une analyse génétique, 24 souches particulièrement typiques de la littérature.

Voici les conclusions essentielles que nous pouvons en tirer:

1. L'hérédité dominante monogénique est confirmée.

2. La sex-ratio dans les familles atteintes est de 61,2 hommes pour 38,8 femmes. femmes.

3. La proportion des sexes dans les familles saines, est de 38 hommes pour 43

4. L'expressivité de la maladie est variable. La pénétrance qui est incomplète, est différente selon le sexe. Dans notre matériel sélectionné, le taux de pénétrance s'éleve à $93,6 \%$ chez les hommes, et $42,9 \%$ chez les femmes.

5. Les hommes transmettent le gène dans $68 \%$, et les femmes dans $32 \%$ des cas.

6. Le sexe-linkage incomplet de la maladie peut être exclu.

7. Nous comparons les valeurs réellement observées avec les taux théoriques d'hommes et de femmes normaux et atteints, pour l'éventualité d'une dominance autosomique. Ces observations permettent de conclure qu'il se forme trop peu de femmes tarées, en comparaison des femmes saines et des hommes atteints.

8. Nous avancons une nouvelle théorie du mécanisme héréditaire, en nous basant sur les différentes valeurs statistiques:

Le facteur responsable serait létal pour le $60 \%$ des zygotes XX tarés. Mais le $60 \%$ des gamètes $\mathrm{X}$ tarés produits par la mère, vu qu'ils sont atteints d'une incompatibilité essentielle pour le spermatozoïde $X$ sain, seraient fécondés électivement par un gamète $Y$. Ce mécanisme nous explique l'excédent d'hommes malades, la faible proportion de femmes atteintes, et la sex-ratio de $61,2: 38,8$.

La concordance des valeurs observées et des taux théoriques prévus pour un mécanisme de ce genre, semble confirmer l'exactitude de notre nouvelle théorie.

\section{Bibliographie}

ADAMs, W.: Brit. med. J., 1878, 928.

Apert, M.: Soc. méd. Hôp., 1925, Réf. Zbl. Chir. 1926, 1678.

Bunch, J. L.: Brit. Dermat., 25, 279, 1913.

CASPARI: Arch. orthop. Heilk., 1896, 143.

Coenen, H.: a) Erg. Chir., 10, 1171, 1918. b) Med. Klin. 1935, II, 1657.

Couch, H.: Canada M. A. J., 39, 225, 1938.

Czoersz: Klin. Wschr., 1928, I, 236.

DebrunNer, H.: Z. orthop. Chir., 62, 321, 1934. 
Deckner, K.: a) Zbl. Chir., 1937, 119. b) Zbl. Chir. 1938, 1192, c) Z. schrift für Menschliche Vererb. u. Konstitut. lehre, 22, 734, 1939.

Doberauer: Beitr. klin. Chir., 36, 123, 1902.

Ebstein, W.: Dtsch. Arch. klin. Med., 103, 201, 1911.

Espinosa Valdes, M.: Bol. Soc. cubanan derm. sif., 5, 1C5, 1948; Réf. Curr. List. Med. Lit., 16, $18,1949$.

Fairbank, H. A. T.: Proc. roy. Soc. Med., 26, 103, 1932.

Franceschetti, A.: Arch. Jul. Klaus-Stiftg., 17, 473, 1942.

Goyrand, D’Aix: Mém. Acad. roy. méd. Paris, 3, 489, 1833.

Hellmuller, H.: Etiologies et pathogénie de la maladie de Dupuytren, Thèse, Genève, 1948.

JaErisch, M.: Ueber Dupuytren'sche Fingerkontraktur, Diss. Halle, 1898.

Jentsch, F. R.: Erbarzt 1937, N. 6, 85.

Jalavisto, E.: Ann. Chir. Gynaec. Fenn., 41, 182, 1952.

KanAvel, Koch et MASON : Surg. etc., 48, 145, 1929.

KARTSCHIKJAN : Z. orthop. Chir., 48, 36, 1927.

KEEN, W. W.: Philadelphia Med. Times, 12, 1881.

Kinzel, H.: Dupuytren'sche Fingerkontraktur, Diss. Breslau, 1927.

Kocher, Th.: Zbl. Chir., 26 et 27, 481 et 497, 1887.

Krogrus, ArI : a) Zbl. Chir., 47, 914, 1920. b) Acta chir. scand. (Stockh), 54, 33, 1922.

LoEwY, J.: Zbl. inn. Med., 1913, 51.

Maurer, G.: Dtsch. Z. Chir., 246, 685, 1936.

Meyerding, H. W.: Arch. Surg., 32, 320, 1936.

Oehlecker, F.: Beitr. klin. Chir., 149, 333, 1930.

SACHS: Derm. Zbl. Hautkrkh., 24, 746, 1927.

SchNITZLER: Münch. med. Wschr., 1935 I, 248.

Schroeder, C. H.: a) Zbl. Chir., 1933, 2214. b) Zbl. Chir., 1934, 1056. c) Arch. orthop. Chir., 35, 125, 1934. d) Dtsch. Z. Chir., 1934, 244. e) Arch. Rassenbiol., 28, 353, 1934.

Schubert, A.: a) Dtsch. Z. Chir., 1923, 177. b) Med. Klin., 1927, I 549.

Sprogis, G.: Dtsch. Z. Chir., 194, 259, 1926.

StaCKebrandT, H.: Die Heredität bei der Dupuytren'schen Kontraktur, dargestellt an 5 Stammbäumen, Diss. Münster, 1932.

Stephensen, S. H. A.: Brit. med. J., 14, 537, 1885.

Then Berg, H.: Allg. Ztschr. f. Psychiat., 112, 327, 1939.

Tord SKoog: Acta chir. scand., 96, suppl. 139, 1948, 1 vol., 190 p.

Ventura, V. E. et Pozzi, P.: Arch. psicol. neurol., 13, 146, 1952 (Milan); Réf. Curr. List. Med. Lit., $22,655,1952$.

Vizıoli: Giorn. Neuropat., 4, 1, 1886; Réf. Zbl. Neur., 1887, 58.

Vogt, A.: Klin. Mbl. f. Augenhlk., 100, 497, 1938.

Vogt, A., Wagner, H., Richner, H. et Meyer, G.: Arch. Jul. Klaus-Stiftg., 14, 475, 1939.

WeBEr, E.: Ztschr. f. Altersforsch., 3, 79, 1941.

Nous exprimons tous nos sentiments de gratitude à Monsieur A. Kaelin, math. dipl. de I'EPF à Zürich. Il a bien voulu expertiser la partie statistique. 


\section{RIASSUNTO}

La malattia di Dupuytren è più frequente di quanto generalmente non si creda. Vengono sottoposti ad analisi 24 tipici alberi ricavati dalla letteratura.

Le conclusioni essenziali che possono essere tirate sono le seguenti :

I) L'eredità dominante monogena è confermata.

2) Il rapporto dei sessi nelle famiglie colpite è di $6 \mathrm{I}, 2$ maschi per 38,8 femmine.

3) La proporzione dei sessi nelle famiglie sane è di $38 \mathrm{ma}-$ schi per 43 femmine.

4) L'espressività della malattia è variabile. La penetranza, che è

\section{SUMMARY}

Dupuytren's disease recurs more frequently than generally assumed. 24 typical family trees derived from the literature are analyzed here.

The main conclusions that can be derived are the following:

I) Monogenic heredity is confirmed.

2) The sex ratio in the affected families is $61,2 \mathrm{~m}: 38,8 \mathrm{f}$.

3) The sex ratio in unaffected families is $38 \mathrm{~m}: 43 \mathrm{f}$.

4) The expressivity of the disease is variable. Penetrance, which is incomplete, varies according to the sex. In our selected material, the penetrance incompleta, è diversa secondo il sesso. Nel nostro materiale selezionato, il tasso di penetranza è di $93,6 \%$ presso i maschi e di 42,9\% presso le femmine.

5) I maschi trasmettono il gene nel $68 \%$ e le femmine nel $32 \%$ dei casi.

6) Si può escludere il linkage incompleto al sesso.

7) Vengono comparati i valori realmente osservati con i tassi teorici di uomini e donne normali e colpiti per l'eventualità di una dominanza autosomica. Queste osservazioni permettono di concludere che il numero delle femmine tarate è troppo basso in confronto delle femmine sane e dei maschi tarati.

rate is $93,6 \%$ in the males and $42,9 \%$ in the females.

5) Males transmit the gene in $68 \%$ of the cases; females in $32 \%$.

6) Incomplete sex linkage can be excluded.

7) A comparison is made between the really observed values and the theoretical rates for normal and affected men and women for the chance of an autosomatic dominance. These observations make possible the conclusion that the number of affected females is too low as compared with unaffected females and affected males.

8) A new theory is suggested concerning the mechanism
8) Viene avanzata una nuova teoria circa il meccanismo ereditario fondandosi sui differenti valori statistici. Il fattore responsabile sarebbe letale per il $60 \%$ dei zigoti XX tarati. Ma il $60 \%$ dei gameti $\mathrm{X}$ tarati prodotti dalla madre, poichè sono affetti da un'incompatibilità essenziale per lo spermatozoo X sano, sarebbero fecondati elettivamente $\mathrm{da}$ un gamete $Y$. Questo meccanismo ci spiega l'eccedenza di uomini ammalati, la debole proporzione di donne colpite e la sex-ratio di $6 \mathrm{I}, 2: 38,8$.

La concordanza dei valori osservati e dei tassi teorici previsti per un meccanismo di questo genere, sembra confermare l'esattezza della nostra nuova teoria.

of heredity based on the different statistical values. The responsible factor would be deadly for $60 \%$ of the XX affected zygotes. But $60 \%$ of the affected $\mathrm{X}$ gametes produced by the mother, being identified by an essential incompatibility for the unaffected $X$ spermatozoid, would be electively fecundated by a $\mathrm{Y}$ gamate. This machanism would explain the excess of affected males, the small proportion of affected females and the sex-ratio of $61,2: 38,8$.

The concordance between the observed values and the theoretically expected values of such a kind seem to convalidate our new theory. 


\section{ZUSAMMENFASSUNG}

Die Dupuytren'sche Krankheit ist häufiger als allgemein angenommen wird.

Es werden 24 besonders typische Sippen und Familien der Literatur erbbiologisch untersucht. Wir ziehen daraus folgende Schlussfolgerungen:

I) Der dominante, monogene Erbgang der Krankheit wird bestätigt.

2) Das Geschlechtsverhältnis in den befallenen Familien beträgt $61,2 \%$ Männer zu $38,8 \%$ Frauen.

3) In den nicht befallenen Familien, beträgt die Sex-Ratio $38: 43$.

4) Der Ausdrucksgrad der Krankheit ist variabel. Deren Penetranz ist unvollständig und geschlechtsverschieden. In unserem ausgelesenen Material, be- trägt der Penetranzgrad $93,6 \%$ bei den Männern und $42,9 \%$ bei den Frauen.

5) Männer vererben das Krankheitsgen in $68 \%$, und Frauen in $32 \%$ der Fälle.

6) Ein unvollständig geschlechtsgekoppelter Erbgang kann ausgeschlossen werden.

7) In der Annahme es handle sich um einem dominanten autosomalen Erbgang, vergleichen wir die praktisch gefundenen Werte mit den theoretisch zu erwartenden, in Bezug auf die Zahl der gesunden und behafteten Frauen und Männer. Aus diesen Untersuchungen geht hervor dass der prozentuale Anteil kranker Frauen zu klein ist, im Vergleich mit den gesunden Frauen und den befallenen Männern.

8) Die statistisch nachgewiese- nen Werte erlauben uns eine neue Theorie des Erbmechanismus aufzustellen:

Das Krankheitsgen würde sich bei $60 \%$ der behafteten XX-Zygoten als Letalfaktor auswirken. - $60 \%$ der von der Mutter gebildeten, kranken X-Gameten hätten aber eine primäre Abneigung für das gesunde X-Spermatozoon, und würden elektiv durch eine Y-Geschlechtszelle befruchtet. Dieser Mechanismus erklärt uns den Ueberschuss an kranken Männern, die kleine Zahl befallener Frauen, sowie das Geschlechtsverhältnis von $6 \mathrm{I}, 2: 38,8$.

Die Konkordanz der beobachteten Werte mit den, für einen solchen Erbmechanismus zu erwartenden, theoretischen Zahlen, scheint die Richtigkeit unserer Theorie zu bestätigen. 